\title{
Gut responses tamed by friendly bacteria
}

We usually live in harmony with tens of millions of bacteria in our gut and would be less healthy without them. Indeed, an imbalance in the normal commensal microbiota is thought to contribute to the development of inflammatory bowel diseases. Now, a study in Nature shows that a factor produced by Bacteroides fragilis, a common commensal bacterium in mammals, can prevent intestinal inflammation in mice.

In this study, the authors first made use of a well-established model of experimental colitis that

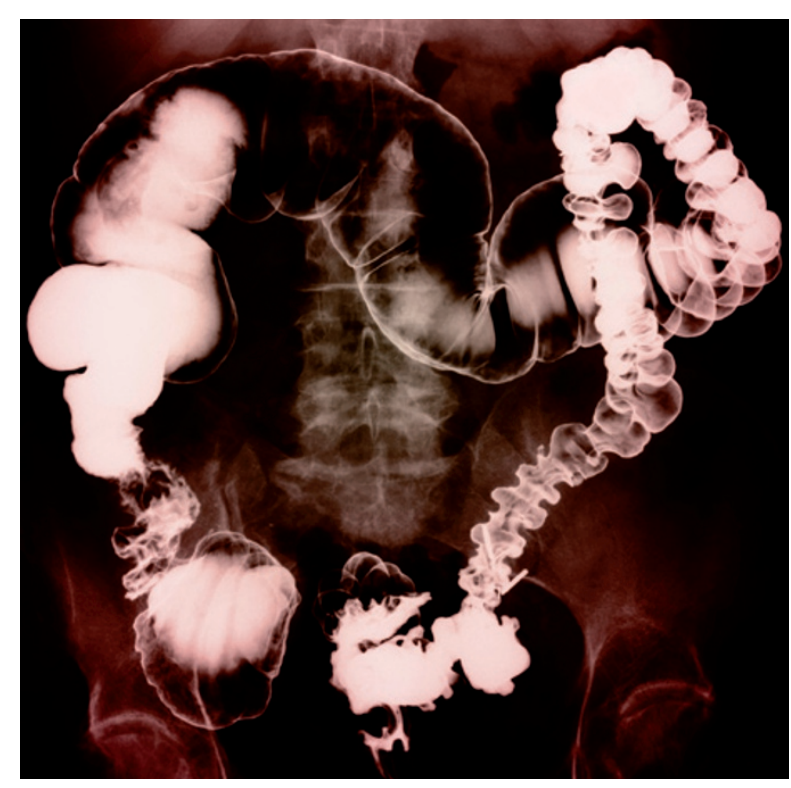

involves the transfer of 'pathogenic' $\mathrm{CD} 4{ }^{+} \mathrm{CD} 45 \mathrm{RB}^{\text {hi }} \mathrm{T}$ cells into specific pathogen-free immunodeficient mice. Following cell transfer, the mice were colonized with Helicobacter hepaticus, which causes severe colitis in the immunocompromised animals. However, co-colonization with $B$. fragilis protected the mice from disease; intestinal tissues from these mice did not show increased levels of the pro-inflammatory cytokines tumour-necrosis factor (TNF), interleukin-1 $\beta$ (IL-1 $\beta$ ) and IL-12p40, and the animals did not suffer from an inability to gain weight. This protective effect did not occur, however, if the mice received a mutant strain of $B$. fragilis that is defective in its ability to produce polysaccharide A (PSA), suggesting a role for this microbial factor in the inhibition of colitis.

The beneficial effect of PSA was then confirmed by showing that oral administration of purified PSA was sufficient to almost completely protect mice from colitis induced by $\mathrm{CD}^{+} \mathrm{CD} 45 \mathrm{RB}^{\text {hi }} \mathrm{T}$-cell transfer and $H$. hepaticus colonization. Studies in a second model of experimental colitis, induced by rectal administration of trinitrobenzene sulphonic acid, also supported the protective effect of PSA. In this model, oral PSA prevented the induction of the disease-causing $\mathrm{T}$ helper 17 cells and elevated TNF production.

Next, the authors investigated the mechanism of PSA-mediated protection against colitis. They showed that $\mathrm{CD} 4^{+} \mathrm{T}$ cells purified from the mesenteric lymph nodes of PSA-treated mice contained higher levels of mRNA encoding the anti-inflammatory cytokine IL-10. Consistent with a role for IL-10 in PSA-mediated protection, administration of IL-10-receptor-specific antibody during oral treatment with PSA abrogated protection from colitis induced by T-cell transfer and H. hepaticus colonization. Finally, IL-10 production by the transferred $\mathrm{T}$ cells was shown to be required for protection, as mice receiving $\mathrm{CD}^{+}{ }^{+} \mathrm{CD} 45 \mathrm{RB}^{\text {hi }} \mathrm{T}$ cells from $\mathrm{Il10^{-/- }}$ mice could not be protected against $H$. hepaticus-induced disease with PSA treatment.

Together, these results show that factors from so-called friendly bacteria might have a crucial role in maintaining our harmonious relationship with commensal bacteria.

Lucy Bird

ORIGINAL RESEARCH PAPER Mazmanian, S. K. Round, J. L. \& Kasper, D. L. A microbial symbiosis factor prevents intestinal inflammatory disease. Nature 453, 620-625 (2008)

FURTHER READING Kullberg, M. C. Soothing intestinal sugars. Nature 453, 602-604 (2008) 